\title{
TOWARDS A MATHEMATICAL UNDERSTANDING OF COLONIZATION RESISTANCE
}

\section{A PREPRINT}

\author{
Erida Gjini* \\ Center for Computational and Stochastic Mathematics \\ Instituto Superior Tecnico, University of Lisbon \\ Lisbon, Portugal \\ erida.gjini@tecnico.ulisboa.pt
}

\author{
Sten Madec \\ Institut Denis Poisson \\ University of Tours, \\ Tours,France \\ Sten.Madec@lmpt . univ-tours.fr
}

January 17, 2021

\begin{abstract}
Microbial community composition and dynamics are key to health and disease. Explaining the forces generating and shaping diversity in the microbial consortia making up our body's defenses is a major aim of current research in microbiology. For this, tractable models are needed, that bridge the gap between observations of patterns and underlying mechanisms. While most microbial dynamics models are based on the Lotka-Volterra framework, we still do not have an analytic notion of colonization resistance, by which a microbial system's fitness as a whole can be understood. Here, we propose a modeling framework where similar species interact with each other at the micro-scale through a co-colonization interaction network encompassing competition and cooperation. This model is based on a generic notion of shared resources between species, affords explicit mathematical results for frequency-dependent dynamics among $N$ species, and offers a precise definition of colonization resistance, directly related to Fisher's fundamental theorem. We contend this approach can be a powerful new tool to model, test and validate interaction networks in complex microbial consortia, and quantify their role in colonization resistance and system invasibility.
\end{abstract}

Keywords colonization resistance model $\cdot$ replicator equation · pairwise invasion fitness network $\cdot$ system invasibility $\cdot$ multispecies community

\section{Introduction}

The human microbiota plays a crucial role in health and disease. Recent studies are increasingly uncovering one of its clearest contributions: protection against invading pathogens, known as colonization resistance [3]. This protection is important when enteric bacterial pathogens challenge the gatrointestinal tract, but applies also in the nasopharynx, skin, and other environments which are populated by rich consortia of commensal microbes. The composition of gut microbiota affects several collective metabolic functions and feedbacks with the host's immune system, leading to or sometimes impairing immune homeostasis. Disruption of healthy microbiota, for example through antibiotics, or cytotoxic chemotherapy, can result in loss of colonization resistance and increased susceptibility to pathogens [2, 25], as well as long-term shifts in microbial ecosystem composition [15]. Conversely, reconstitution of normal microbiota (e.g. through faecal transplants) has been demonstrated to restore host protection, and help cure patients from recurring pathogenic infections [29]. Such dynamic processes of loss and gain of colonization resistance are particularly important in the intestinal tract, making stability, diversity and resilience of our gut microbial ecosystem an intense topic of investigation [20, 18, 27].

Despite the progress in experimentally revealing the beneficial roles of the $>100$ species (trillions of microbes) populating the human intestine [16], a theoretical understanding of how colonization resistance emerges as a collective trait from a community network, is maintained as a dynamic process throughout lifetime, and reacts to various

\footnotetext{
${ }^{*}$ Corresponding author
} 
A PREPRINT - JANUARY 17, 2021

perturbations, remains elusive. Mathematical approaches so far have been based primarily on Lotka-Volterra models [28, 8, 24] to explore dynamics and stability of intestinal microbiota. However, a mathematical definition and understanding of colonization resistance is still lacking. Explicit frameworks are needed for new perspectives on perturbation effects on the system, such as antibiotic administration, immune suppression or host interaction with other drugs and vaccines. Here, we synthesize a new conceptual framework to describe colonization resistance at the level of a single host, developing an analogy with an epidemiological multi-strain system [21]. Application of this model to in-host microbiota dynamics requires re-interpretation of original variables and parameters, and a shift of scales.

\section{A host is a system of 'free' and 'occupied' micro-niches that can be colonized}

We define the host as the system, and the dimensionality of the system as the number of similar microbial types (entities) interacting in colonization of such a system, hereafter denoted as 'species'. In the re-framing following [21], we model the within-host environment as a number of potential micro-'niches' to be filled, and we track the proportion of such niches that remain free or susceptible $(S)$, those that get singly-colonized by either species $\left(I_{i}\right)$, and those that get co-colonized, either twice by the same species $\left(I_{i i}\right)$ or by two different species $\left(I_{i j}\right)$ (Figure 1 1 A). By taking suitably small niche units within-host, we can truncate the multiplicity of 'infection' (MOI) to at most 2 species per unit.

Microbial propagation dynamics, assuming homogeneous mixing of propagules in the in-host milieu, can be described, to a first-order approximation through simple mass action kinetics (Figure 11B). The growth of each microbial species depends on their transmission rate $\beta$ and frequency of niches 'emitting' propagules of that species. The transmission parameter, $\beta$, in this analogy, can be taken as a net parameter, encapsulating the chain of events including local growth happening in the micro-niche of 'origin', followed by migration, and diffusion of infectious propagules in the system, multiplied by the effective probability of settling in other 'destination' niches. In its basic version, the model assumes $\beta$ is equal for all $i$, yielding reproductive fairness in the system.

Competition among species happens upon co-colonization, where the colonizer $(i)$ and co-colonizer $(j)$ microbes may compete or facilitate each other, each pair with coefficient $K_{i j}$. Even without specifying the mechanisms (crossfeeding/antagonism/bacteriocins), this implies that the micro-scale presence of one species alters the value of that same resource or spatial unit for individuals of that same/other species. Such altered susceptibilities $K_{i j}$ ( $>1$ : facilitation, $<1$ : competition), for $N=2$, form a 2-by-2 matrix (Figure $1 \mathrm{C}$ ), but in general are $N$-by- $N$. Singly- and co-colonized patches produce equal number of propagules. Mixed co-colonization patches $I_{i j}$ produce and transmit $i / j$ propagules with probability $1 / 2$.

Free units of resource are replenished at rate $r$, assumed equal to the natural death rate, keeping thus constant the carrying capacity. Colonization episodes are cleared at rate $\gamma$, yielding a turnover rate of $m=r+\gamma$. The basic reproduction number $R_{0}$, denotes how many new colonizations, each colonized niche will produce over its lifetime as occupied, if occurring in a totally colonization-free environment, and is given by $\beta / m$, similar to epidemic models: if $R_{0}>1$ there is a globally stable endemic state with $1-1 / R_{0}$ colonization level. If $R_{0}<1$, the system is colonization-free. With this established analogy, the $N$-species model [21] can be applied to microbiota dynamics within host, as a first-order approximation, in much the same spirit as a Lotka-Volterra dynamic model, but with the unit of resource made explicit.

\section{Explicit in-host frequency dynamics of $N$ species under homogeneous mixing}

Assuming phenotypic similarity in micro-scale interaction trait space between species ( $K_{i j}=k+\epsilon \alpha_{i j}$, with $\epsilon$ small), we have shown for $N=2$ and for general $N$ in [21], that there are two timescales in such a system: a fast one where the species follow neutral dynamics, and global colonization and co-colonization variables stabilize, and a slow timescale, where non-neutral dynamics happen, driven explicitly by the co-colonization interaction matrix $K$. Applying the similarity assumption in $N$ dimensions [21] (where $k$ can reflect the mean of $K_{i j}$, and $\epsilon$ their standard deviation), we have mapped the $N$ - species dynamics explicitly to a special replicator equation [13] for the change in frequencies over long time $\tau=\epsilon t$ :

$$
\frac{d}{d \tau} z_{i}=\Theta z_{i} \cdot\left(\sum_{j \neq i} \lambda_{i}^{j} z_{j}-Q(\mathbf{z})\right), \quad i=1, \cdots, N
$$

with $\sum_{i} z_{i}=1$ and $\lambda_{i}^{j}$ denoting mutual invasion fitnesses between species (Table 1 . The mutual invasion fitness, a key quantity at the heart of adaptive dynamics [11], in our model is given by: $\lambda_{i}^{j}=\alpha_{j i}-\alpha_{j j}-\mu\left(\alpha_{i j}-\alpha_{j i}\right)$, and is a direct function of the phenotypic variation in co-colonization interactions (the $\alpha_{i j}$ 's), and of the ratio of single to co-colonization in the system, $\mu$. Together, the $\lambda_{i}^{j}$ between all pairs, drive collective dynamics. The parameter $\Theta$ 
A PREPRINT - JANUARY 17, 2021

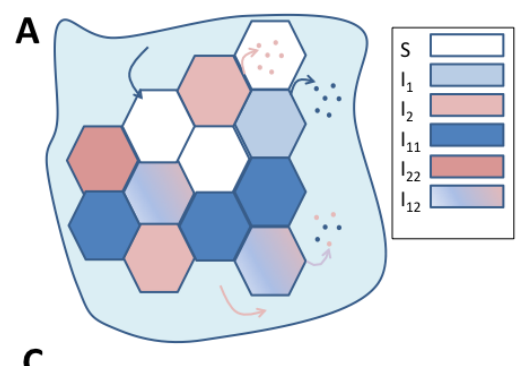

B

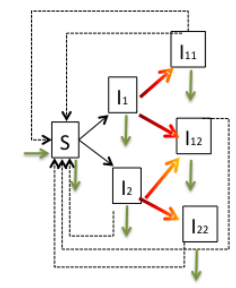

C
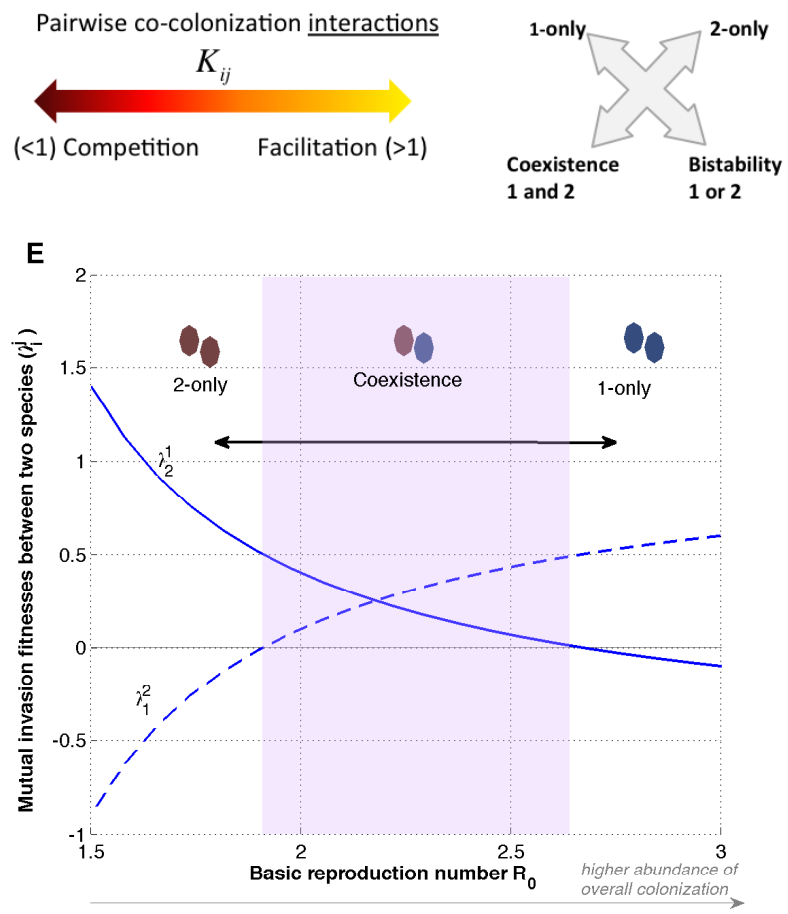

Figure 1: Analogy between epidemiological and within-host multi-species dynamics $($ here $N=2)$. The model is based on SIS dynamics with co-infection [21]. A. Conceptualization of the within-host environment, where micro-niches can be free (uncolonized $S$ ), singly- $\left(I_{i}\right)$ or doubly-colonized $\left(I_{i i}, I_{i j}, I_{j j}\right)$. Full equations are given in [21]. B. Model structure for niche state transitions, including colonization (black arrows), co-colonization (color-shaded arrows) and clearance (dashed arrows). Natural birth/death is denoted by green arrows. C. Singly-colonized patches may have a reduced or increased susceptibility to becoming co-colonized, noted by coefficients $K_{i j}(>/<1)$ between any two species. D. For $N=2$, there are four outcomes (Table 1), depending on species interactions: exclusion, coexistence, or bistability. D. Context-dependence of mutual invasion fitnesses $\left(\lambda_{i}^{j}\right)$ generates different ecology between 2 species $\left(K_{i j}\right.$ fixed). Starting from coexistence, lowering overall colonization $\left(R_{0} \downarrow\right)$, eg. via antibiotics, or increasing it drastically, may lead to competitive exclusion favouring an opposite species in each direction.

determines the speed of selection. Finally, the dynamic term $Q$ gives colonization resistance in the system:

$$
Q(\mathbf{z})=\sum_{1 \leq k<j \leq N}\left(\lambda_{j}^{k}+\lambda_{k}^{j}\right) z_{j} z_{k} .
$$

It sums the global effect of the system on each member, and reflects mathematically the mean invasion fitness of the multi-species consortium [21]. Because $Q$ involves a product of frequencies, it is non-zero, only when there are more than 1 species coexisting. In the simplest system, $N=2$ (Table 1), it is easy to see that at the coexistence steady state, $Q$ is positive and maximized for $z_{1}=z_{2}=1 / 2$. Thus, a 2 -species system is mostly protected against a third invader, when its members are equiabundant. 
A PREPRINT - JANUARY 17, 2021

\section{Table 1: Key features of the co-colonization model for similar species [21]}

\section{The constituent variables and parameters are the following:}

$\begin{array}{ll}\text { Micro-scale co-colonization interactions }(N \times N) & K_{i j}=k+\epsilon \alpha_{i j} \text { (similarity assumption) } \\ \text { Rescaled interaction coefficients } & \alpha_{i j}=\frac{K_{i j}-k}{\epsilon} ;\left(k=\text { mean of } K_{i j}, 0 \leq \epsilon \ll 1\right) \\ \text { Basic reproduction number } & R_{0} \\ \text { Equilibrium colonization prevalence }\left(I^{*}+D^{*}\right) & 1-S^{*}=1-1 / R_{0} \\ \text { Ratio of single to co-colonization }\left(I^{*} / D^{*}\right) & \mu=\left[\left(R_{0}-1\right) k\right]^{-1} \\ \text { Invasion fitness of } i \text { in a } j \text {-only equilibrium } & \lambda_{i}^{j}=\alpha_{j i}-\alpha_{j j}-\mu\left(\alpha_{i j}-\alpha_{j i}\right) \text { (pairwise) } \\ \text { Speed of selection } & \Theta=\beta\left(1-\frac{1}{R_{0}}\right)\left(\frac{\mu}{2(\mu+1)^{2}-\mu}\right) . \\ \text { Timescale of selection (slow timescale) } & \tau=\epsilon t \\ \text { Frequency of species } i & z_{i}(\tau), \text { with } \sum z_{i}=1, i=1, \cdots, N \\ \text { Single/co-colonization prevalence } i /(i, j) & I_{i}=I^{*} z_{i} ; I_{i j}=D^{*} z_{i} z_{j}\end{array}$

Frequency dynamics for $N=2$ is given by:

$$
\frac{d}{d \tau} z_{2}=\Theta z_{2} \cdot\left[\lambda_{2}^{1}\left(1-z_{2}\right)-\left(\lambda_{2}^{1}+\lambda_{1}^{2}\right) z_{2}\left(1-z_{2}\right)\right] z_{1}=1-z_{2}
$$

4 mutual invasion structures $(N=2)$

\section{4 final outcomes $(N=2)$}

$$
\begin{aligned}
& \lambda_{1}^{2}>0, \lambda_{2}^{1}<0 \\
& \lambda_{1}^{2}<0, \lambda_{2}^{1}>0 \\
& \lambda_{1}^{2}>0, \lambda_{2}^{1}>0 \\
& \lambda_{1}^{2}<0, \lambda_{2}^{1}<0
\end{aligned}
$$

1 wins in competitive exclusion: $z_{1}^{*}=1, z_{2}^{*}=0$

2 wins in competitive exclusion: $z_{1}^{*}=0, z_{2}^{*}=1$

1 and 2 coexist: $z_{1}^{*}=\lambda_{1}^{2} /\left(\lambda_{2}^{1}+\lambda_{1}^{2}\right), z_{2}^{*}=1-z_{1}^{*}$

Bistability: $z_{1}^{*}=1$, or $z_{2}^{*}=1$ depending on $z_{1}(0)$ 'priority effect'

\section{Effect on parameters}

$$
\begin{aligned}
& R_{0} \uparrow\left(R_{0} \downarrow\right) \\
& R_{0} \uparrow \\
& k \downarrow(\text { or } k \uparrow) \\
& \text { Different } K_{i j} \text { entries }
\end{aligned}
$$

The system with $N$ interacting species evolves according to:

$$
\frac{d}{d \tau} z_{i}=\Theta z_{i} \cdot\left(\sum_{j \neq i} \lambda_{i}^{j} z_{j}-\sum_{1 \leq k \neq j \leq N} \sum_{j} \lambda_{j}^{k} z_{j} z_{k}\right), \quad i=1, \cdots, N
$$

\section{Colonization resistance as mean invasibility of the system}

$$
Q(\mathbf{z})=\sum \sum_{1 \leq k \neq j \leq N} \lambda_{j}^{k} z_{j} z_{k}
$$

Colonization resistance $Q$ captures the essential tension between individual and group success in the system, as species dynamically shape their common environment.

- If $Q>0$, the system as a whole acts negatively on each species, making $Q$ a cost to any current member of the consortium, but by consequence, affecting negatively also invaders, thus a net benefit for the external stability of the system.

- If $Q<0$, the system as a whole acts positively on all species, increasing their growth rate, but also facilitating invaders. 
A PREPRINT - JANUARY 17, 2021
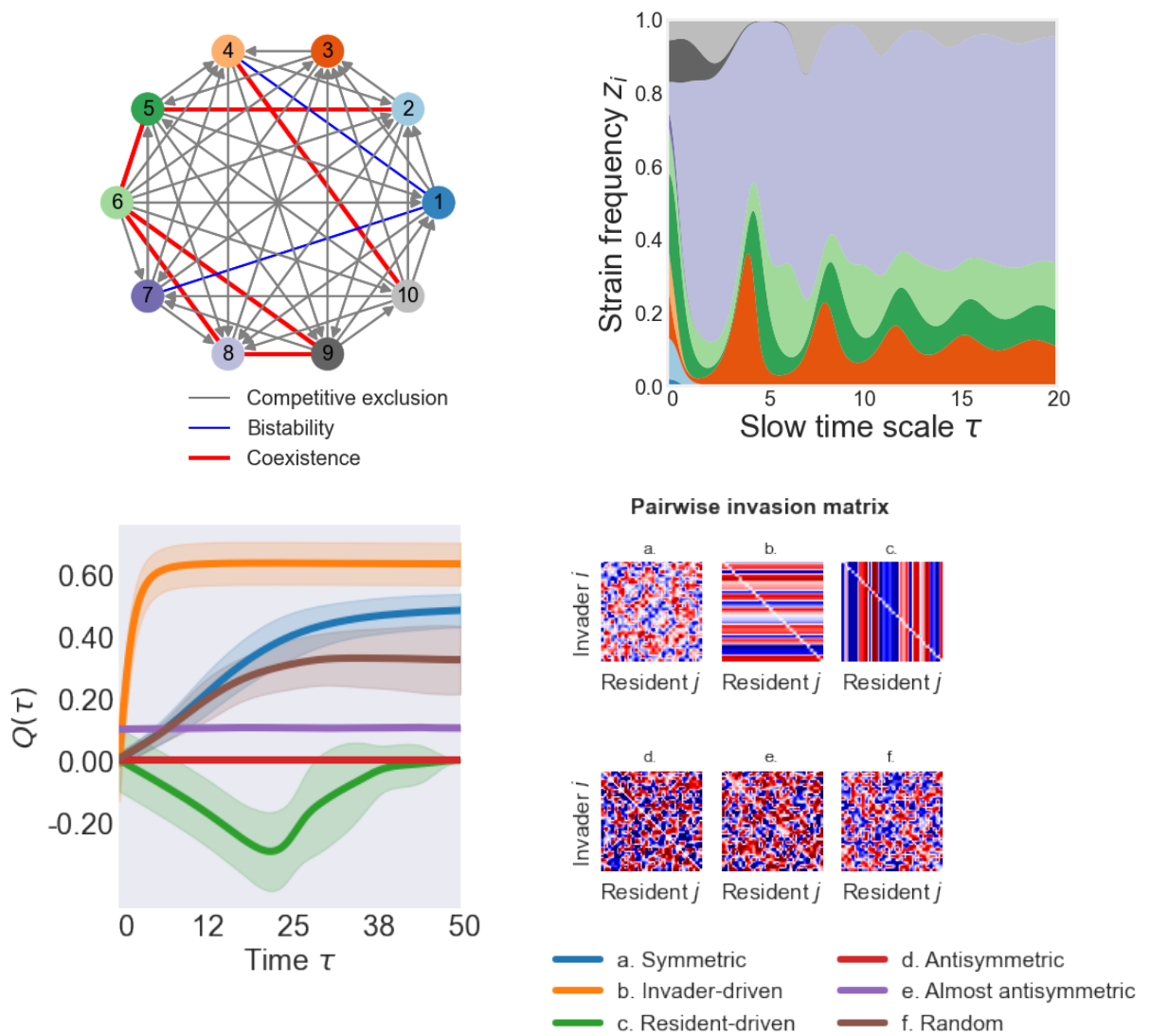

Figure 2: Mutual invasion, dynamics and system mean invasion fitness. Top panel: Illustration of a mutual invasion network between 10 species, whose structure and pairwise invasion fitness values lead to multispecies dynamics on the right $(\mathrm{Eq} 1$, with ultimately 5 species coexisting. Bottom panel: Colonization resistance for special invasion structures. On the left, we plot $Q$ dynamics (mean: lines, \pm sd.: shading) over 30 stochastic realizations for 6 cases of $\Lambda$ between $N=30$ species (shown on the right). Random uniform $\lambda_{i}^{j} \in[-1,1]$, are fed into Eqs $1(\Theta=1)$, solved with random $z_{i}(0)$, as in [21]. Symmetric $\Lambda$ yields dynamics described by Fisher's fundamental theorem. Invader-driven $\Lambda$ implies large coexistence potential. Resident-driven $\Lambda$ implies large potential for exclusion. Antisymmetric $\Lambda$ makes $Q \equiv 0$. Almost-antisymmetric $\Lambda$ produces complex dynamics (e.g. limit cycles) leading to periodic $Q$. Random mutual invasion allows rich multi-species dynamics. Cases a-c can be obtained for 3 particular instances of symmetric co-colonization interactions matrix $K[21]$.

\section{Colonization resistance: system vs. outsider species}

Depending on how many species make up the resident consortium, and how they interact, one models a different system size $N$, a network of mutual invasion fitnesses (edges), frequency dynamics, and ultimately system resilience to invasion by outsiders (Table 1 1 . The micro-scale effects between species upon co-colonization are captured by the $K_{i j}$ which denote a fixed pairwise interaction trait. The mutual invasion fitnesses between species, $\lambda_{i}^{j}$, emerge as higher-level and context-dependent traits [5], in our case determined by $R_{0}, k$. It is the $\lambda_{i}^{j}$ that ultimately govern relative species abundances, co-occurrence, diversity, stability, and system properties (see Movies S1-S2). Pairwise $i-j$ coexistence for $N=2$ does not imply $i-j$ coexistence in a community with more species.

For symmetric mutual invasion $\left(\lambda_{i}^{j}=\lambda_{j}^{i}\right), Q$ always increases, and this case corresponds to Fisher's fundamental theorem [9]. For anti-symmetric mutual invasion $\left(\lambda_{i}^{j}=-\lambda_{j}^{i}\right), Q=0$, corresponding to zero-sum games [6]. Dynamics in that case tend to a (structurally unstable) family of cycles around a center for an odd number of species, like in Lotka-Volterra prey-predator models [1]. More special cases and explicit $K-\Lambda$ matrix links are analyzed in [21]. Keeping all else equal, $Q$ increases fastest for invader-driven mutual invasion (Fig. 2 ). 
A PREPRINT - JANUARY 17, 2021

General structures of co-colonization interactions between $N$ species yield coexistence scenarios of more complex nature than fixed points, including limit cycles and chaos [12]. Thus, depending on how frequency dynamics unfold, subject to intrinsic or extrinsic drivers, $Q$ dynamics can be higher or lower, monotonic or oscillatory, and feed back on the system, in a fully analytically-explicit manner (Table 1 .

\subsection{Antibiotics can change colonization resistance and effect depends on species interactions}

It is precisely the analytical tractability of this model [21, 12] that makes it easier to study global perturbation effects, and to interpolate across conditions (Table 1 ). Perturbations affecting global context, such as $R_{0}$ and $k$, directly change the mutual invasion fitnesses $\lambda_{i}^{j}$, and thus species dynamics. An example are broad-spectrum antibiotics, leading to alteration of $R_{0}$ in the system, likely reducing the colonization of all species, and shifting their internal competitive balance. Even when acting symmetrically on two members, more antibiotics can drive the system from coexistence to competitive exclusion (Fig. 1E.), hence toward $Q=0$. Through our explicit $\lambda_{i}^{j}$ [21], one can clearly see that this $R_{0}$ impact drastically varies, depending on the interaction network between species, for example it is stronger when $k$ is lower, i.e. when competition is higher in the system (see Movies S3-S4). In particular, even when starting at same baseline invasion fitnesses between two species (i.e. also the same coexistence scenario in terms of relative abundances), the stronger the mean competition in micro-scale co-colonization (the lower $k$ ), the more drastic will be the selection in the system induced by the antibiotic. Such explicit individualization of perturbation effects, based on underlying interactions, may be easily related to data and to context-dependence of outcomes.

In more diverse communities, perturbation effects are undoubtedly more complex. Among $N$ species, a key modulator of coexistence regimes is the ratio of single-to co-colonization, $\mu$ [12], decreasing with $R_{0}$ and $k$. When $\mu \rightarrow \infty$, and all else fixed, the mutual invasibility network contains mostly competitive exclusion edges, which makes more species coexist, but in unstable and oscillatory fashion [12]. Thus, decreasing $R_{0}$, leading possibly to a fluctuating $Q$, can make a given system more vulnerable to opportunistic invasion, particularly when $Q$ is low or negative, even if transiently.

\subsection{Invasion by outsiders depends on colonization resistance}

Mathematically, it is straightforward to see that the initial growth rate of the invader in a system at state $\mathbf{z}(\tau)$ is given by:

$$
r_{\text {invader }}=\sum_{j \in \text { system }} \lambda_{\text {invader }}^{j} z_{j}-Q(\mathbf{z})
$$

The first summation term in $r_{\text {invader }}$ depends on invader traits, $\lambda_{\text {invader }}^{j}$, namely how this species invades any existing species currently in the system. The second term instead, $-Q$, is independent of the invader, and describes how the system colonization resistance by itself, detracts from invader's initial growth, if $Q>0$.

We have verified this numerically with model simulations. When comparing different multi-species systems in their resistance to invasion, for fixed parameters, we find that the higher the value of $Q$, the harder the invasion by outsider species (Fig. 3A-G). In contrast, we find no significant role of system diversity (Shannon, Gini-Simpson indices Fig 3 [-K), but a positive effect of the number of species for invader success (Fig. 3 $\mathrm{H}$ ). The final outcome of any given invasion however, as captured in $\mathrm{Eq} 3$, besides system $Q$, depends also on other details of resident dynamics, and crucially, on the traits of the invader, relative to the community, at the invasion time.

\section{Discussion}

Colonization resistance in microbiology [18] is closely linked with the theory of invasibility in ecology, long studied but rarely integrated between the disciplines. Elton argued that complex communities should be more resistant to invasion by new species [17]. Later May [22, 23] showed that complex ecological communities tend to be less stable, i.e. in these systems it is harder to return to equilibria from small perturbations to existing species. Since then, the complexity-stability debate began, inspiring many investigations [19]. Crucial to study invasibility and stability of ecosystems have been simulations, using random interaction networks or varying their properties, e.g. the percentage of cooperative vs. competitive links [26].

While until now, no unifying analytical quantity has emerged, two notions of stability, internal vs. external, have been studied, with the latter receiving much less attention. It is external stability that relates most with colonization resistance. While the stabilizing vs. destabilizing role of mutualism is still theoretically debated [26], as are diversity effects on external stability [10], recent work is attempting to reconcile Elton's and May's views [14] and calling for deeper and more concise mechanistic insight. 

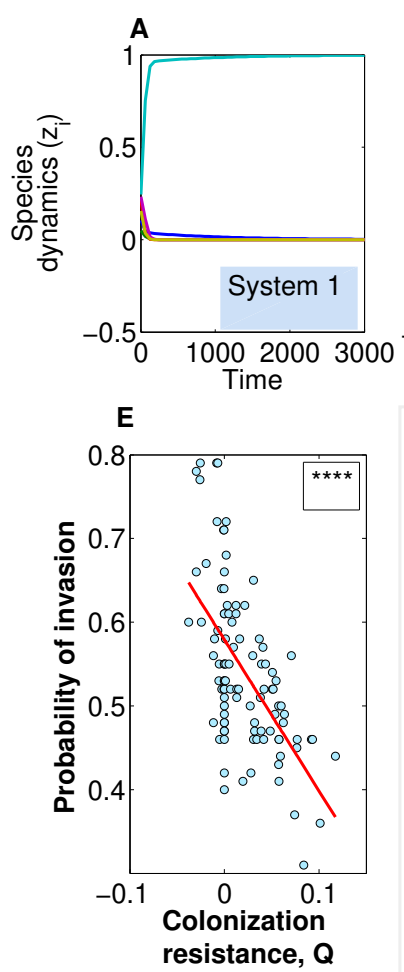
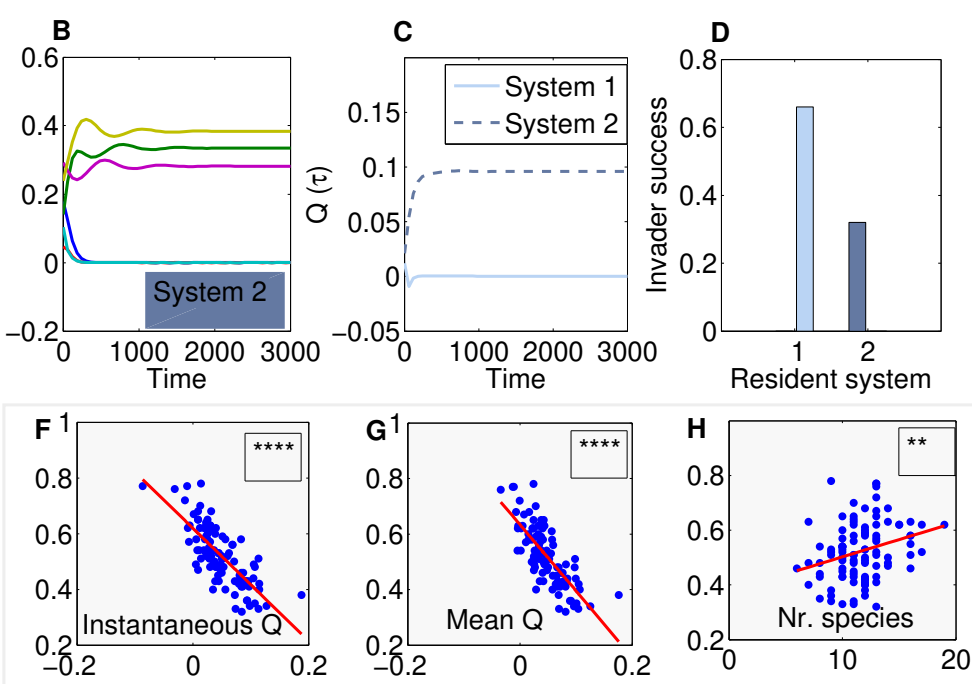

$\mathrm{N}=20$
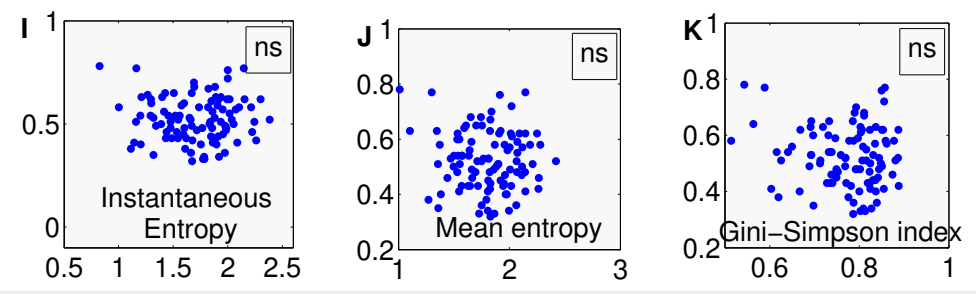

Figure 3: Colonization resistance and outsider invasion. In A-D, we fixed the $K_{i j}$ interactions in co-colonization in two systems with $N=6, \beta=1, k=0.3, R_{0}=2$. We simulated 100 realizations of invasion by an outsider species, sampling its interaction traits from the same distribution as the resident $\mathcal{N}\left(k, \epsilon^{2}\right)$, and introducing it at the mid-point of the interval. We counted invasion successful if the invader grew from its low initial frequency $\left(10^{-4}\right)$. System 2 (higher $Q$ ) was superior in preventing invasion to system 1 . In $\mathbf{E}$, we repeated the procedure for 100 randomly generated systems (blue dots) and examined system $Q$ at invasion and invader success, finding a significant negative relationship between colonization resistance and invasibility $\left(p<10^{-5}\right)$. The final outcome of invasion whether the invader ultimately coexists, or how original species are affected was not studied. In $\mathbf{F}-\mathbf{K}$, we examined invasion success for $N=20$. Simulating 100 random systems as in $\mathbf{E}$, we regressed outsider invasion probability on different system characteristics: $Q\left(p<10^{-5}\right)$ in $(\mathbf{F}-\mathbf{G})$ and the number of species in the system $(p<0.01)$ in $(\mathbf{H})$ were significant, while diversity indices (I-K) were not.

In this brief report we offer a fresh conceptual framework to bridge between these fields and contribute to the complexitystability debate. We propose unification between ecological, evolutionary dynamics and emergent system properties in multi-species communities. Our derived replicator equation for multi-species dynamics, obtained from an analogy with an epidemiological model, simplifies complexity, while directly linking with Lotka-Volterra models [4] and with the ecological theory of invasibility [19, 7].

Many theoretical phenomena, like those explored above and beyond, directly accessible via this formalism, could be empirically matched when comparing gradients in microbiota dynamics caused by antibiotics or other stressors in multi-species communities. These can include variation across human or mammalian hosts of different age or conditions, or environments under the effect of abiotic factors such as moisture, light, temperature (e.g. microbial consortia in soil, cheese rinds, natural biofilms). With model extensions to increase realism and generality under way (unpublished results), we anticipate the backbone of the replicator equation formalism to stay the same, where the precise notion of colonization resistance can inform deeper biological understanding and more precise links with data.

In light of increasing calls for models to better describe microbial consortia, here we outline a new path for explicit mathematical study of colonization resistance and mean fitness in a microbial ecosystem. This framework may help synthesize a new understanding of the ecology, development, and evolution of host health and resilience to disease, mediated by microbiota. 
A PREPRINT - JANUARY 17, 2021

\section{References}

[1] Stefano Allesina and Jonathan M. Levine. A competitive network theory of species diversity. Proceedings of the National Academy of Sciences, 108(14):5638-5642, 2011.

[2] Carolyn D Alonso, Suzanne B Treadway, David B Hanna, Carol Ann Huff, Dionissios Neofytos, Karen C Carroll, and Kieren A Marr. Epidemiology and outcomes of clostridium difficile infections in hematopoietic stem cell transplant recipients. Clinical infectious diseases, 54(8):1053-1063, 2012.

[3] Marjorie Bohnhoff, Barbara L Drake, and C Phillip Miller. Effect of streptomycin on susceptibility of intestinal tract to experimental salmonella infection. Proceedings of the Society for Experimental Biology and Medicine, 86(1):132-137, 1954.

[4] Immanuel M Bomze. Lotka-volterra equation and replicator dynamics: new issues in classification. Biological cybernetics, 72(5):447-453, 1995.

[5] Scott A Chamberlain, Judith L Bronstein, and Jennifer A Rudgers. How context dependent are species interactions? Ecology letters, 17(7):881-890, 2014.

[6] Tsuyoshi Chawanya and Kei Tokita. Large-dimensional replicator equations with antisymmetric random interactions. Journal of the Physical Society of Japan, 71(2):429-431, 2002.

[7] Charles Sutherland Elton. The ecology of invasions by animals and plants. Springer Nature, 2020.

[8] Karoline Faust and Jeroen Raes. Microbial interactions: from networks to models. Nature Reviews Microbiology, 10(8):538-550, 2012.

[9] Ronald Aylmer Fisher. The genetical theory of natural selection. The Clarendon Press, 1958.

[10] Jason D Fridley, John J Stachowicz, S Naeem, DF Sax, EW Seabloom, MD Smith, TJ Stohlgren, D Tilman, and B Von Holle. The invasion paradox: reconciling pattern and process in species invasions. Ecology, 88(1):3-17, 2007.

[11] Stefan AH Geritz, Géza Mesze, Johan AJ Metz, et al. Evolutionarily singular strategies and the adaptive growth and branching of the evolutionary tree. Evolutionary ecology, 12(1):35-57, 1998.

[12] Erida Gjini and Sten Madec. The ratio of single to co-colonization is key to complexity in interacting systems with multiple strains. biorxiv preprint: 10.1101/2020.02.03.932806 (accepted Jan.2021), 2020.

[13] Josef Hofbauer and Karl Sigmund. Evolutionary game dynamics. Bulletin of the American Mathematical Society, 40(4):479-519, 2003.

[14] Cang Hui and David M Richardson. How to invade an ecological network. Trends in ecology \& evolution, 34(2):121-131, 2019.

[15] Cecilia Jernberg, Sonja Löfmark, Charlotta Edlund, and Janet K Jansson. Long-term ecological impacts of antibiotic administration on the human intestinal microbiota. The ISME journal, 1(1):56, 2007.

[16] Sohn Kim, April Covington, and Eric G Pamer. The intestinal microbiota: antibiotics, colonization resistance, and enteric pathogens. Immunological reviews, 279(1):90-105, 2017.

[17] Roger L Kitching. A world of thought:?the ecology of invasions by animals and plants? and charles elton's life's work. Fifty Years of Invasion Ecology: The Legacy of Charles Elton, pages 1-10, 2010.

[18] Trevor D Lawley and Alan W Walker. Intestinal colonization resistance. Immunology, 138(1):1-11, 2013.

[19] Jonathan M Levine and Carla M D'Antonio. Elton revisited: a review of evidence linking diversity and invasibility. oikos, pages 15-26, 1999.

[20] Catherine A Lozupone, Jesse I Stombaugh, Jeffrey I Gordon, Janet K Jansson, and Rob Knight. Diversity, stability and resilience of the human gut microbiota. Nature, 489(7415):220, 2012.

[21] Sten Madec and Erida Gjini. Predicting n-strain coexistence from co-colonization interactions: epidemiology meets ecology and the replicator equation. Bulletin of Mathematical Biology, 82(142), 2020.

[22] Robert M May. Will a large complex system be stable? Nature, 238(5364):413-414, 1972.

[23] Robert M May. Stability and complexity in model ecosystems, volume 1. Princeton university press, 2019.

[24] Babak Momeni, Li Xie, and Wenying Shou. Lotka-volterra pairwise modeling fails to capture diverse pairwise microbial interactions. Elife, 6:e25051, 2017.

[25] Robert C Owens Jr, Curtis J Donskey, Robert P Gaynes, Vivian G Loo, and Carlene A Muto. Antimicrobialassociated risk factors for clostridium difficile infection. Clinical Infectious Diseases, 46(Supplement_1):S19-S31, 2008. 
[26] Jimmy J Qian and Erol Akçay. The balance of interaction types determines the assembly and stability of ecological communities. Nature Ecology \& Evolution, 4(3):356-365, 2020.

[27] Ana Sousa, Nelson Frazão, Ricardo S Ramiro, and Isabel Gordo. Evolution of commensal bacteria in the intestinal tract of mice. Current Opinion in Microbiology, 38:114-121, 2017.

[28] Richard R Stein, Vanni Bucci, Nora C Toussaint, Charlie G Buffie, Gunnar Rätsch, Eric G Pamer, Chris Sander, and Joao B Xavier. Ecological modeling from time-series inference: insight into dynamics and stability of intestinal microbiota. PLoS computational biology, 9(12):e1003388, 2013.

[29] Els Van Nood, Anne Vrieze, Max Nieuwdorp, Susana Fuentes, Erwin G Zoetendal, Willem M de Vos, Caroline E Visser, Ed J Kuijper, Joep FWM Bartelsman, Jan GP Tijssen, et al. Duodenal infusion of donor feces for recurrent clostridium difficile. New England Journal of Medicine, 368(5):407-415, 2013. 
A PREPRINT - JANUARY 17, 2021

\section{Supplementary files}

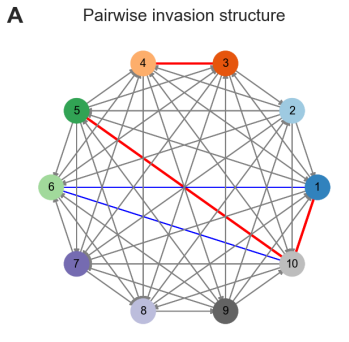

C

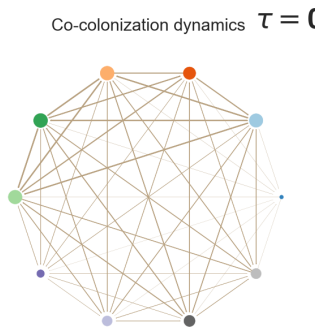

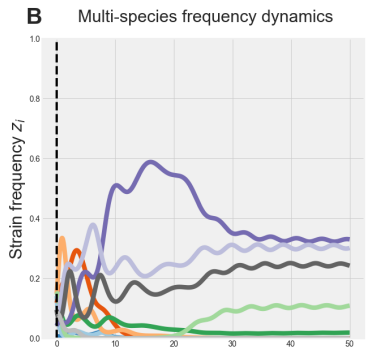

$Q=0.45$

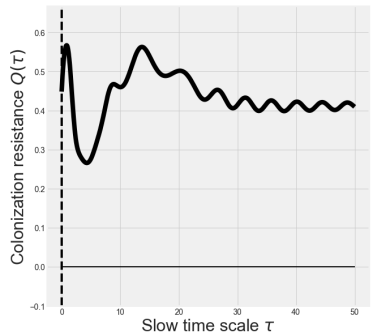

Movie S1 Illustration of model dynamics in co-colonization and species frequency space (example 1). Here we visualize multi-species dynamics and evolution of system invasibility [21], following the derived replicator equation 1. We used randomly generated $K_{i j}, \mu=0.5, k=1, \epsilon=0.1, \beta=2$, and random initial conditions among $N=10$ species. A. Pairwise invasion network structure. In $\mathbf{B}$, we show the evolution of relative abundances over time. In $\mathbf{C}$, the width of the nodes is scaled according to the frequency of each species $\left(z_{i}\right)$, the width of the edges between any two species is scaled according to their product $\left(z_{i} z_{j}\right)$. D Mean invasion fitness $Q$ displays mainly positive values over time, indicating high resistance to invasion of this system.

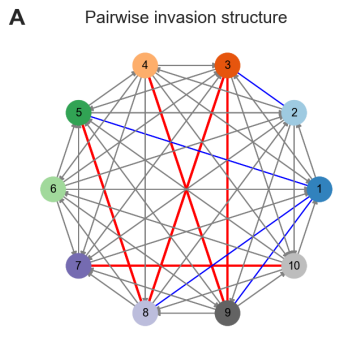

C

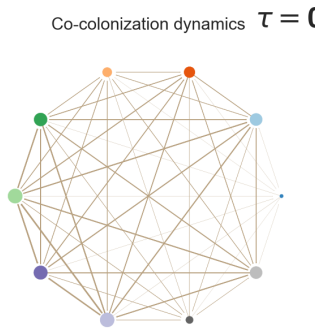

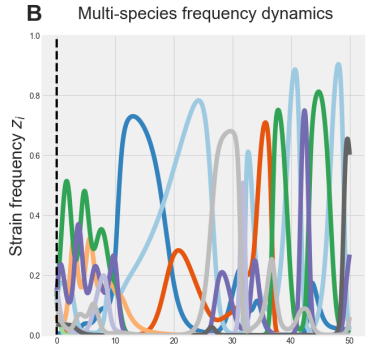

$Q=0.26$

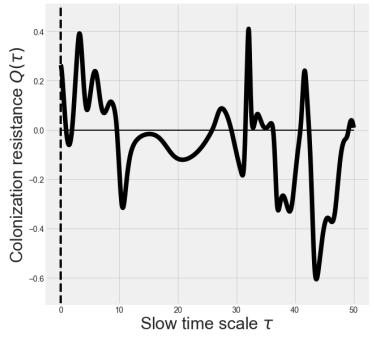

Movie S2 Illustration of model dynamics in co-colonization and species frequency space (example 2). Here we visualize multi-species dynamics and evolution of system mean invasibility, following Equation 1 , with another set of randomly generated $K_{i j}, \mu=0.5, k=1, \epsilon=0.1, \beta=2$ and random initial conditions among $N=10$ species. A. Pairwise invasion network structure. In $\mathbf{B}$, we show the evolution of relative abundances over time. In $\mathbf{C}$, the width of the nodes is scaled according to the frequency of each species $\left(z_{i}\right)$, the width of the edges between any two species is scaled according to their product $\left(z_{i} z_{j}\right)$. D Colonization resistance $Q$ displays wild oscillations over time, spanning the positive and negative range, indicating transient windows of time where this system favours outsider species invasion. 
A PREPRINT - JANUARY 17, 2021
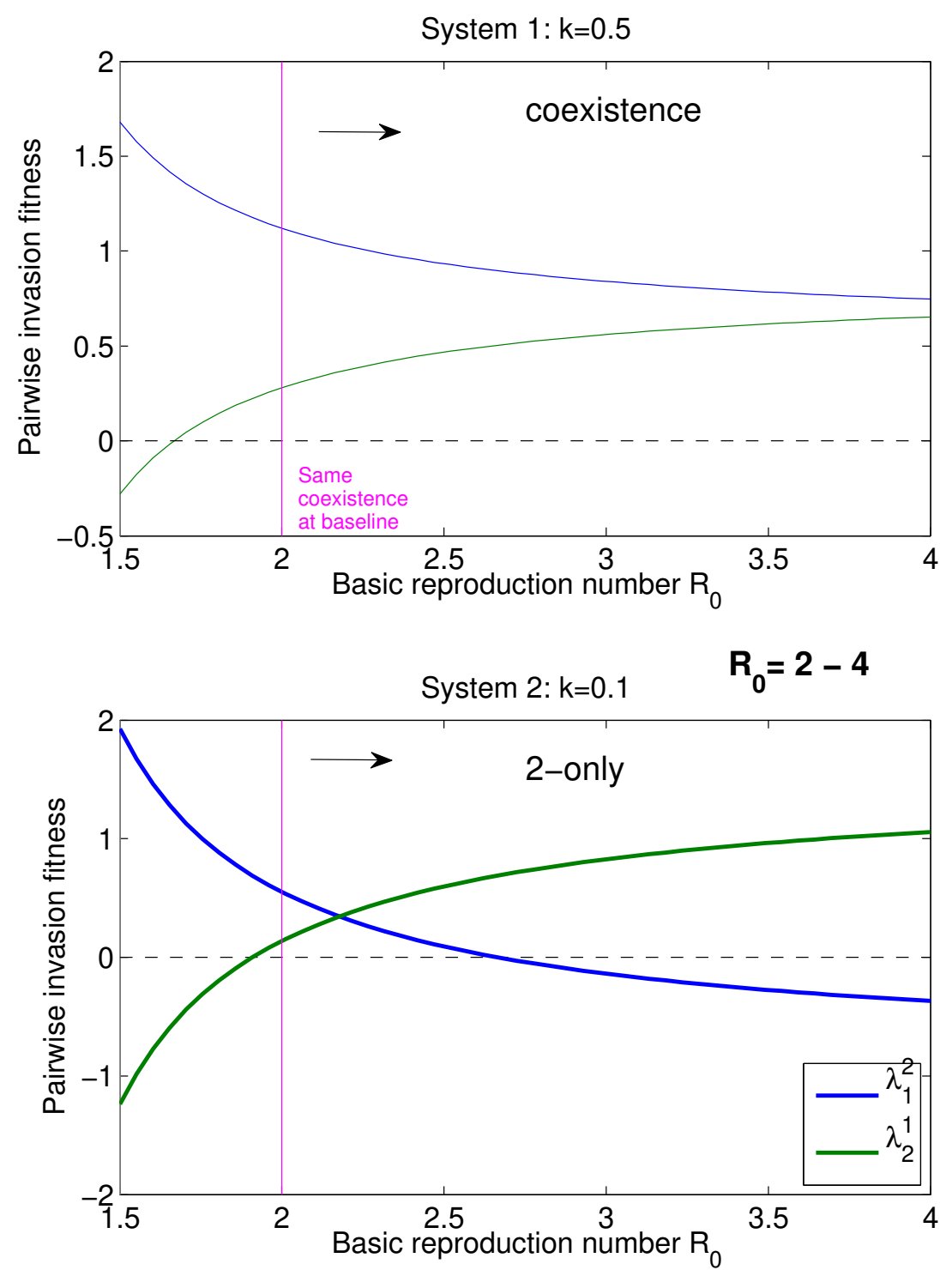

Movie S3 Increasing basic reproduction number $R_{0}$ has system-specific effects on the outcome between two interacting species. Here we visualize the context-dependence of species outcomes for $N=2$, via $\lambda_{i}^{j}$ as a function of basic reproduction number $R_{0}$ (scaling the overall prevalence of colonization according to $1-1 / R_{0}$ and the ratio of single to co-colonization $\mu=1 /\left(k\left(R_{0}-1\right)\right)$ ). We compare two systems (1 and 2) which vary in primary $K_{i j}$ co-colonization interactions, but display same equilibrium frequencies $\left(z_{1}, z_{2}\right)$ between two species coexisting at $R_{0}=2$ (magenta line). Increasing $R_{0}$ has very different effects on the two systems. While system 1 (less competitive, higher $k$ ) preserves coexistence, system 2 (more competitive, lower $k$ ), for the same changes in $R_{0}$ shifts towards exclusion. This shows that perturbation effects depend explicitly on underlying species network. The rescaled interaction coefficients are as follows, for system 1: $\alpha_{11}=0.2801, \alpha_{12}=1.1202, \alpha_{21}=1.4003, \alpha_{22}=0.8402$ and for system 2 : $\alpha_{11}=-0.9638, \alpha_{12}=0.5507, \alpha_{21}=0.6884, \alpha_{22}=1.5145$. 
A PREPRINT - JANUARY 17, 2021
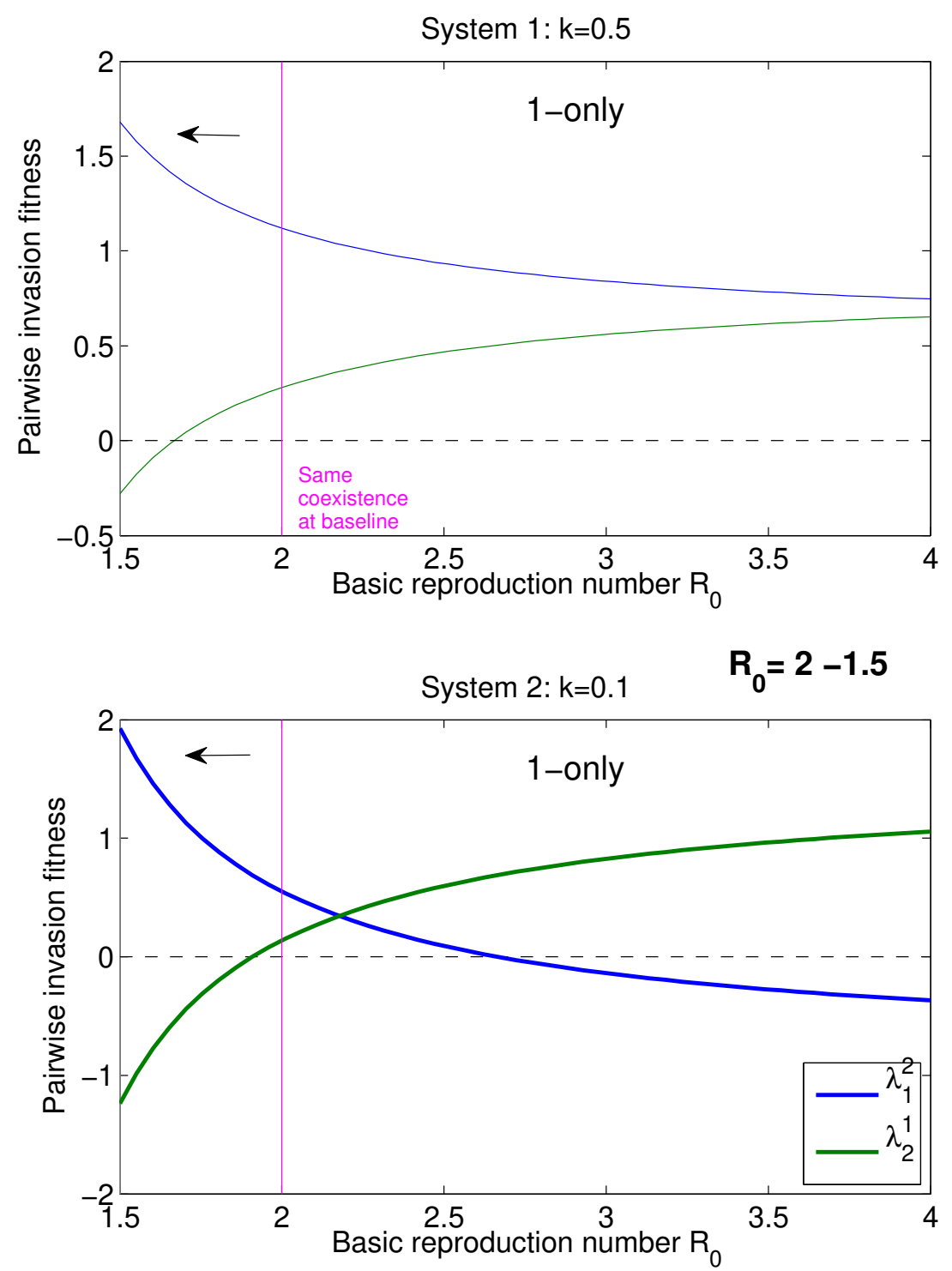

Movie S4 Decreasing basic reproduction number $R_{0}$ has system-specific effects on the outcome between 2 interacting species. Here we visualize the context-dependence of species mutual invasion fitnesses (ecological outcome) for $N=2$, decreasing net microbial growth intensity $R_{0}$, hence reducing the overall prevalence of colonization according to $1-1 / R_{0}$. We compare two systems (1 and 2 ) which vary in $K_{i j}$ co-colonization interactions (system 1 is less competitive than system 2), but display same coexistence between two species at $R_{0}=2$ (magenta line). Decreasing $R_{0}$ has very different effects on the two systems. While system 1 preserves coexistence for longer, system 2 , for the same changes in $R_{0}$ shifts faster towards exclusion. This highlights that perturbation effects depend explicitly underlying species network. The rescaled interaction coefficients are as in Movie S3. 